\title{
Does the median preoptic nucleus contribute to sympathetic hyperactivity in spontaneously hypertensive rats?
}

\author{
Aline A. Mourão ${ }^{\text {a }}$, Marina C.S. Moreira ${ }^{\mathrm{a}}$, Aryanne B.S. Melo a ${ }^{\mathrm{a}}$, Paulo R. Lopes ${ }^{\mathrm{a}}$, Ana C.S. Rebelo ${ }^{\mathrm{b}}$, Daniel A. Rosa ${ }^{\mathrm{a}}$, \\ André H. Freiria-Oliveira ${ }^{a}$, Eduardo Colombari ${ }^{c}$, Gustavo R. Pedrino ${ }^{\mathrm{a}, *}$ \\ a Center for Neuroscience and Cardiovascular Research, Department of Physiological Sciences, Biological Sciences Institute, Federal University of Goiás, Goiânia, Goiás, Brazil \\ b Department of Morphology, Biological Sciences Institute, Federal University of Goiás, Goiânia, Goiás, Brazil \\ ' Department of Physiology and Pathology, School of Dentistry, São Paulo State University, Araraquara, São Paulo, Brazil
}

\section{A R T I C L E I N F O}

\section{Article history:}

Received 2 October 2015

Received in revised form 8 December 2015

Accepted 16 February 2016

\section{Keywords:}

Arterial pressure

Hypertension

$\mathrm{MnPO}$

Renal sympathetic nerve activity

SHR

\begin{abstract}
A B S T R A C T
The present study sought to determine the involvement of median preoptic nucleus (MnPO) in the regulation of the cardiovascular function and renal sympathetic activity in normotensive (NT) and spontaneously hypertensive rats (SHR). MnPO inhibition evoked by Muscimol ( $4 \mathrm{mM}$ ) nanoinjections, elicited fall in MAP and renal sympathoinhibition in NT-rats. Surprisingly, in SHRs these responses were greater than in NT-rats. These results demonstrated, for the first time that MnPO was involved in the tonic control of sympathetic activity in NT and SHRs. Furthermore, our data suggest the MnPO involvement in the increased sympathetic outflow and consequent arterial hypertension observed in SHRs.
\end{abstract}

(C) 2016 Elsevier B.V. All rights reserved.

\section{Introduction}

Multiple evidences in the literature point to the central nervous system (CNS) participation in the development and maintenance of the hypertension (Blanch et al., 2013; Toney et al., 2010). Moreover, studies have shown evidence of an increase in sympathetic activity in many hypertension models (Oliveira-Sales et al., 2014; Toney et al., 2010). In rats with renovascular hypertension or spontaneously hypertensive rats (SHR), increase in sympathetic tone and plasma concentration of noradrenaline were observed (Linz et al., 2015; Oliveira-Sales et al., 2014). Together these studies suggest direct role of sympathetic nervous system (SNS) in the development and maintenance of this pathology.

Previous experimental investigations demonstrated the involvement of MnPO in the ingestive behavior, endocrine and cardiovascular adjustments induced by acute changes in volume or composition of the extracellular fluid compartment (EFC; (McKinley and Johnson, 2004; Pedrino et al., 2009). Lesion that encompasses the MnPO region reduced sodium $\left(\mathrm{Na}^{+}\right)$ingestion evoked by systemic sodium depletion (De Luca et al., 1992; Gardiner et al., 1986), and vasopressin secretion in response to hyperosmolarity (Mangiapane et al., 1983; McKinley et al., 2004). Overall, these studies illustrated the important role of MnPO in

\footnotetext{
* Corresponding author at: Department of Physiological Science, Universidade Federal de Goiás, Estrada do Campus, s/n., Zip code: 74001-970, PO: 131. Goiânia, GO, Brazil.

E-mail addresses: pedrino@ufg.br, gpedrino@gmail.com (G.R. Pedrino).
}

body fluid homeostasis. However, the participation of $\mathrm{MnPO}$ in the tonic control of SNS in normovolemic condition remains to be clarified. Thus, we tested the hypothesis that, besides the involvement of the $\mathrm{MnPO}$ in reflex responses induced by acute changes in the EFC, this nucleus may also be involved in the tonic autonomic and cardiovascular regulation. Moreover, we investigated the participation of MnPO in the increase in sympathetic nerve activity and arterial blood pressure $(A B P)$ in SHR.

\section{Methods}

\subsection{Animals}

Male Wistar normotensive (NT) rats and SHRs weighing 250-350 g were used. The animals were housed individually in stainless steel cages in a room with controlled temperature $\left(23 \pm 2{ }^{\circ} \mathrm{C}\right)$, water and food ad libitum. Lights were on from 7:00 am to 7:00 pm. The animals were provided by the Universidade Federal de Goiás (UFG). The protocols used in this work were approved by the ethics committee of the UFG (protocol number: 34/12) and performed according to the National Institutes of Health Guide for the Care and Use of Laboratory Animals.

\subsection{Surgical procedures}

The rats were anesthetized with urethane $(1.2 \mathrm{~g} / \mathrm{kg}$, i.v.; SigmaAldrich, St. Louis, MO, USA) and the femoral vein and artery were 
catheterized for drug administration and recording of ABP, respectively. Tracheostomy was performed to reduce airway resistance. The rats were positioned in a stereotaxic apparatus for craniotomy and instrumented for recording the renal sympathetic nerve activity (RSNA).

\section{3. $A B P$ and electrocardiogram recording}

In order to record the ABP, the arterial catheter was connected to a pressure transducer that was attached to a Bridge Amplifier (FE221; ADInstruments, Colorado Springs, CO, USA). The pulsatile ABP was recorded continuously with a PowerLab System (ADInstruments, Colorado Springs, CO, USA). The mean arterial pressure (MAP) was calculated from the pulsatile signal using the LabChart program (Chart v7.3.7, ADInstruments, Colorado Springs, CO, USA).

Analog signals of the electrocardiogram (ECG), obtained through electrodes positioned in the forelimbs, were amplified 1000 times and filtered between 100 and $1000 \mathrm{~Hz}$ (ECG100C; ADInstruments, Colorado Springs, CO, USA). The heart rate (HR) was calculated as instantaneous frequency of the ECG signal (Chart v7.3.7, ADInstruments, Colorado Springs, CO, USA).

\subsection{Renal sympathetic nerve activity recording}

For recording the RSNA, the renal nerve was located with the assistance of the microscope, carefully dissected and placed on a pair of bipolar silver electrodes coupled to amplifier (P511 AC, Grass Technologies; Warwick, USA Bridge). The RSNA was amplified 20,000 times and filtered between 30 and $1000 \mathrm{~Hz}$. To quantify the noise of the signal obtained at the end of the experiment, ganglionic blocker hexamethonium (30 mg/kg, iv., Sigma-Aldrich, St. Louis, MO, USA) was administered. The RSNA signal was rectified and integrated (resetting every $1 \mathrm{~s}$; Chart 7 v7.3.7; ADInstruments, Colorado Springs, CO, USA).

\subsection{Nanoinjections into MnPO}

NT rats and SHRs were placed in ventral decubitus on a stereotaxic apparatus and the craniotomy was performed for positioning of the glass micropipette that was coupled to a syringe forming a pressure nanoinjection system. Then, $100 \mathrm{nl}$ of saline $(\mathrm{NaCl} ; 150 \mathrm{mM})$ and

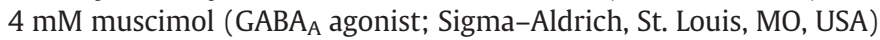
were nanoinjected into $\mathrm{MnPO}$, according to the following coordinates: $0.6 \mathrm{~mm}$ rostral to the bregma, at a depth of $7.1 \mathrm{~mm}$ below the dorsal surface of the brain, modified from: (Paxinos and Watson, 1998). As a negative control, saline $150 \mathrm{mM}$ and $4 \mathrm{mM}$ muscimol (100 nl) were nanoinjected directly into the third ventricle at the following coordinates: $0.0 \mathrm{~mm}$ rostral to the bregma, at a depth of $7.1 \mathrm{~mm}$ below the dorsal surface of the brain. At the end of the experiment, $100 \mathrm{nl}$ of a solution of Evans Blue (4\%; Sigma-Aldrich, St. Louis, MO, USA) were nanoinjected at the same site of previous nanoinjections for further histological confirmation.

\subsection{Histology}

At the end of the experiments the animals were perfused with a $(\mathrm{NaCl} ; 150 \mathrm{mM})$ saline solution, followed by $10 \%$ formaldehyde (LabSynth, Itapira, SP, Brazil). Then, the brain was removed and fixed in the same formaldehyde solution and subsequently stored for a period of $48 \mathrm{~h}$ in $30 \%$ sucrose solution. The brains were dissected into $40 \mu \mathrm{m}$ coronal sections with the aid of a freezing microtome (Leica, Wetzlar, Germany). To determine the sites of nanoinjections into MnPO, the sections obtained from this hypothalamic region were stained using neutral red.

\subsection{Statistical analysis}

Statistical analysis and graph confections were done using GraphPad Prism software (v 5.1). The baseline values were compared between the groups using an unpaired Student's $t$-test. The autonomic and cardiovascular effects induced by saline and muscimol nanoinjections into the MnPO and third ventricle were analyzed by a one-way ANOVA, followed by the Newman-Keuls test. Value of $p<0.05$ was considered statistically significant.

\section{Results}

\subsection{Histological analysis}

Fig. 1A shows photomicrograph of a coronal section of the forebrain of a representative site with $4 \%$ Evans blue nanoinjection. Analysis of the spread of dye nanoinjected at the end of the experiment showed that the drug injection sites were confined to the region that included the MnPO (Fig. 1B).

\subsection{Participation of the median preoptic nucleus in the tonic control of cardiovascular and sympathetic parameters in NT and SHRS}

Both, NT $(n=6)$ and SHR $(n=6)$ exhibited a similar body weight $(278.6 \pm 9.8$ vs. $287.8 \pm 18.7)$ and HR baseline values $(367.5 \pm$ $15.1 \mathrm{bpm}$ vs. $378.2 \pm 15.7 \mathrm{bpm}$ ). The baseline RSNA values of SHR and NT rats were $0.0790 \pm 0.0185$ a.u. and $0.0457 \pm 0.0091$ a.u., respectively. As expected, the baseline MAP in SHR $(124.0 \pm 0.7 \mathrm{~mm} \mathrm{Hg})$ was higher than in NT rats $(108.4 \pm 2.6 \mathrm{~mm} \mathrm{Hg})$.

Nanoinjections of saline (150 mM NaCl) did not change MAP (NT: $\Delta$ $0.4 \pm 0.2 \mathrm{~mm} \mathrm{Hg} / \Delta \% 0.4 \pm 0.2 \%$; SHR: $\Delta 0.2 \pm 0.4 \mathrm{~mm} \mathrm{Hg} / \Delta \% 0.3 \pm$ $0.4 \%$ ), HR (NT: $\Delta 0.7 \pm 0.9 \mathrm{bpm} / \Delta \% 0.2 \pm 0.3 \%$; SHR: $\Delta 1.1 \pm$ $1.4 \mathrm{bpm} / \Delta \% 0.2 \pm 0.4 \%$ ) and RSNA (NT: $\Delta \% 0.4 \pm 0.2 \%$; SHR: $\Delta \%$ $-0.2 \pm 1.3 \%$; Fig. 2A, C, D and E).

The pharmacological inhibition of the MnPO changed the cardiovascular and autonomic parameters in both NT and SHR. Fig. 2A and $\mathrm{B}$ represent typical representative tracings of cardiovascular and autonomic changes caused by nanoinjections into the MnPO of NT and SHR, respectively.

The inhibition of MnPO promoted fall in MAP of NT rats $(\Delta-17.0 \pm$ $1.2 \mathrm{~mm} \mathrm{Hg} / \Delta \%-15.4 \pm 1.1 \%)$. However, this response was strikingly greater $(p<0.05)$ in SHR $(\Delta-31.6 \pm 5.0 \mathrm{~mm} \mathrm{Hg} / \Delta \%-26.8 \pm 3.9 \%$; Fig. $2 \mathrm{~A}, \mathrm{~B}$ and $\mathrm{C}$ ). The range of negative chronotropy in response to muscimol nanoinjections into MnPO were observed in NT $(\Delta$ $-55.4 \pm 12.3 \mathrm{bpm} / \Delta \%-14.3 \pm 3.5 \%$; Fig. $2 \mathrm{~A}$ and $\mathrm{D})$ and in SHR $(\Delta$ $-18.3 \pm 7.2 \mathrm{bpm} / \Delta \%-4.8 \pm 2.0 \%$; Fig. $2 \mathrm{~B}$ e $\mathrm{D})$.

Inhibition of MnPO neurons by muscimol resulted in renal sympathoinhibition in both groups. This pharmacologic blockade resulted in the decrease of RSNA; an effect that elicited greater $(p<0.05)$ impact in SHR $(\Delta \%-54.4 \pm 6.2 \%$; Fig. $2 \mathrm{~B}$ and $\mathrm{E})$ than in NT $(\Delta \%-37.5 \pm 3.94 \%$; Fig. $2 A$ and $E)$.

\subsection{Muscimol nanoinjections into the third ventricle of NT and SHRs}

The saline nanoinjections in third ventricle did not promotes changes in MAP, HR and RSNA $(\Delta-0.2 \pm 0.8 \mathrm{~mm} \mathrm{Hg} / \Delta \%-0.2 \pm 0.8 \%$ vs. $\Delta 1.3 \pm 0.7 \mathrm{~mm} \mathrm{Hg} / \Delta \% 1.1 \pm 0.6 \% ; \Delta 0.7 \pm 0.3 \mathrm{bpm} / \Delta \% 0.2 \pm 0.1 \%$ vs. $\Delta-0.1 \pm 2.4 \mathrm{bpm} / \Delta \% 0.2 \pm 0.9 \% ; \Delta \% 1.7 \pm 0.1 \% v s . \Delta \% 1.6 \pm 0.4 \%)$ in NT $(n=4)$ and SHR $(n=4)$, respectively. Muscimol nanoinjections into the third ventricle did not modify any parameters in NT(MAP: $\Delta$ $0.5 \pm 1.6 \mathrm{~mm} \mathrm{Hg} / \Delta \% 0.4 \pm 1.5 \% ; \mathrm{HR}: \Delta 1.4 \pm 1.5 \mathrm{bpm} / \Delta \% 0.5 \pm 0.5 \%$; RSNA $\Delta \% 0.5 \pm 0.8 \%$; ) and SHR (MAP: $\Delta-1.8 \pm 0.4 \mathrm{~mm} \mathrm{Hg} / \Delta \%$ $-1.3 \pm 0.3 \%$; HR: $\Delta-0.4 \pm 2.1 \mathrm{bpm} / \Delta \%-0.1 \pm 0.6 \%$; RSNA: $\Delta \%$ $-1.6 \pm 1.0 \%)$. These results demonstrate that the responses observed were not mediated by unspecific action of muscimol in neuronal structures around third ventricle. 

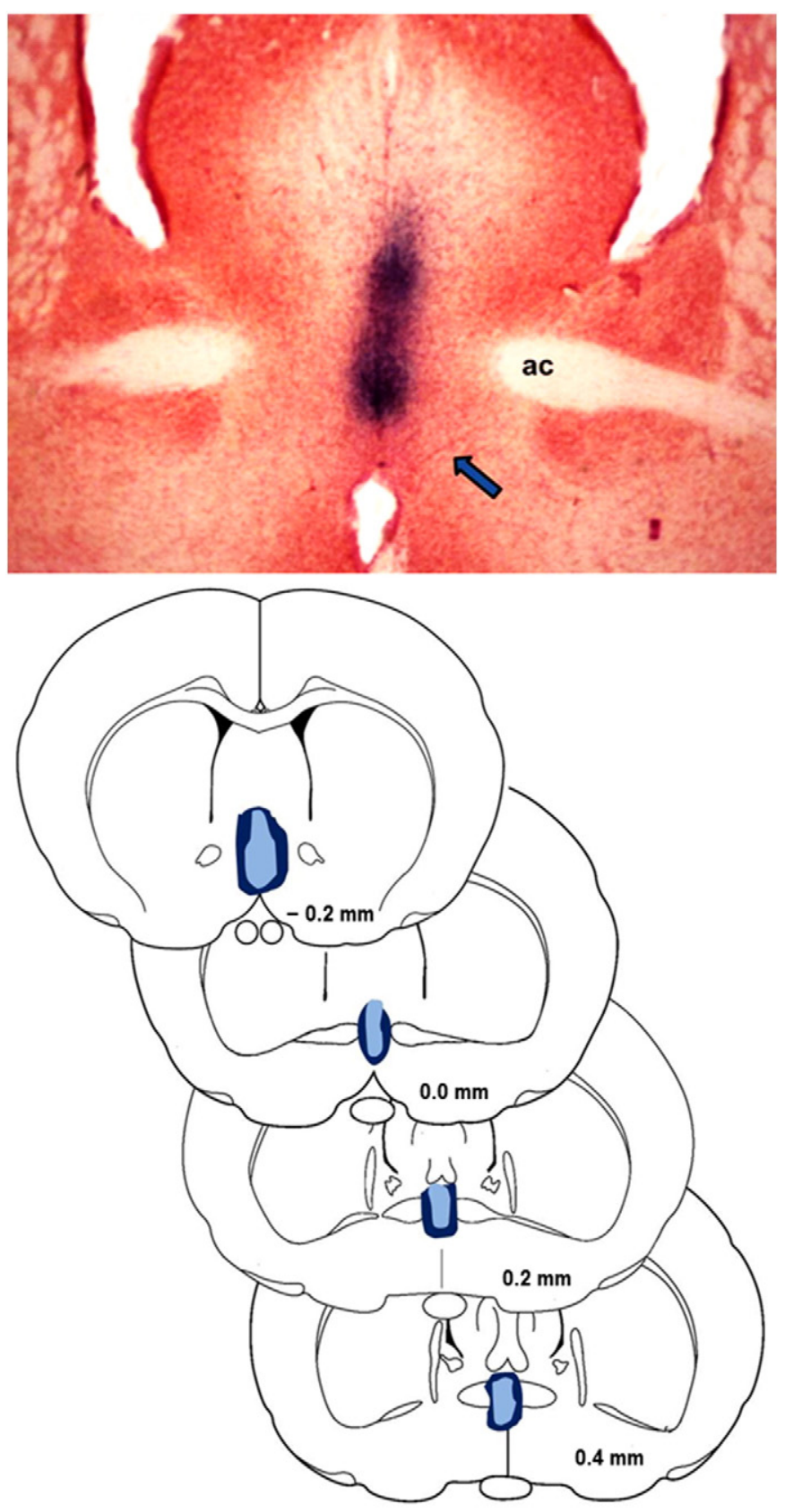

Fig. 1. (A) Photomicrograph of a coronal brain section from one representative rat, showing the nanoinjection site into the Median Preoptic Nucleus (MnPO; arrow). ac, anterior commissure. (B) Four sequential coronal sections showing the approximate spreading of the drugs nanoinjections into the MnPO. Dark blue area: maximal spreading. Light blue area: minimum spreading.

\section{Discussion}

The MnPO have been described as an integral part of the blood fluid and endocrine control (Llewellyn et al., 2012; Pedrino et al., 2009; Stocker and Toney, 2005). Despite evidences highlighting the importance of the MnPO in hydroelectrolytic balance, the participation of this nucleus in tonic regulation of ABP and sympathetic nerve activity remains unknown. In the present study, we have demonstrated the key role performed by the MnPO in the RSNA tonic control and sympathetic hyperactivity observed in SHR. Therefore, these results strongly indicate that the MnPO is a supramedullary area responsible for maintaining the renal sympathetic hyperactivity and consequent hypertension observed in SHR.
The renal territory is crucial to the hydroelectrolytic balance as the kidneys are involved in corporal excretion of excess plasma sodium (Antunes-Rodrigues et al., 2004; Brody and Johnson, 1980; Pedrino et al., 2008; Toney and Stocker, 2010; Yasuda et al., 2000; Da Silva et al., 2013). The renal blood flow is thinly regulated by the renal sympathetic nerves and. Hormonal factors such as atrial natriuretic peptide, oxytocin and vasopressin (Amaral et al., 2014; Pedrino et al., 2008).

Based on limited electrophysiological and anatomical experiments, studies have demonstrated that the involvement of MnPO in cardiovascular and endocrine regulation is due to its inputs to magnocellular and parvocellular regions of the paraventricular nucleus (PVN) (Llewellyn et al., 2012; Stocker and Toney, 2005). Recently, Llewellyn et al. (2012) demonstrated that that the activation of MnPO increases the activity of PVN neurons. Taken together, these results indicate that the $\mathrm{MnPO}$ could be a source of excitatory inputs to the sympathetic premotor neurons of the PVN (Llewellyn et al., 2012).

Hence, it is conceivable that MnPO could modulate, through its PVN connections, the pathways that are involved in sympathetic outflow, and consequently the cardiovascular function. Based on these evidences (Allen, 2002; Takeda et al., 1991), it is conceivable that the inhibition of MnPO could decrease the activity of PVN and RVLM neurons which subsequently result in decreases in the RSNA and MAP. In fact, in the present study, we observed that the inhibition of the MnPO caused decrease in the RSNA and BP in NT rats. Moreover, we also observed that inhibition of MnPO potentiated hypotension and renal sympathoinhibition in SHR, indicate hyperactivity of the nucleus in this hypertension model. To the best of our knowledge, no other study has demonstrated that $\mathrm{MnPO}$ could play a central role in the increase of RSNA and consequent hypertension observed in SHR.

Recent investigations from our laboratory indicating the participation of the MnPO in the ABP control. Silveira et al. (2014) showed that the blockaded of MnPO promoted hypotension in NT rats. Consistent with these results, we have demonstrated that inhibition of the MnPO promotes hypotension in both normotensive and hypertensive rats. Moreover, the present study has advanced on current knowledge, demonstrating, for the first time in the literature, that the inhibition of MnPO induces renal sympathoinhibition, thereby indicating the involvement of this nucleus in the tonic regulation of sympathetic nerve activity.

The MnPO also receives afferences of the regions that participate in cardiovascular modulation, such as catecholaminergic neurons of the Nucleus of the Solitary Tract (NTS; A2 neurons) and ventrolateral medulla ( $A 1$ and $C 1$ neurons; (Tucker et al., 1987). Moreira et al. (2009) demonstrated the importance of the NTS in the maintenance of hypertension in SHR. In this study, the authors showed that lesion of the commissural NTS and Anteroventral Third Ventricle region (AV3V region) reduced the BP of the SHRs (Moreira et al., 2009). The MnPO receives dense projections from the NTS. Hence, both electrolytic lesions of the NTS and pharmacological blockade of the MnPO promote decrease in MAP (which is more expressive in the hypertensive animals). It is not unreasonable to suppose that the MnPO hyperactivity in SHR originate, at least partly, from the NTS.

In summary, the present results show evidence of MnPO neurons participation in the tonic control of BP, RSNA and consequently, in the maintenance of hypertension in SHR. However, further studies are required to elucidate the pathways and neurotransmitters in the MnPO involved in the control of sympathetic nerve activity.

\section{Financial support}

This work was supported by Fundação de Amparo a Pesquisa do Estado de Goiás (FAPEG) grants 2012/0055431086 (GRP) and 2009/ 10267000352 (GRP) and by Conselho Nacional de Desenvolvimento Científico e Tecnológico (CNPq) grants 483411/2012-4 (GRP) and 447496/2014-0 (GRP). The funders had no role in study design, data collection, analysis, decision to publish or preparation of the manuscript. 


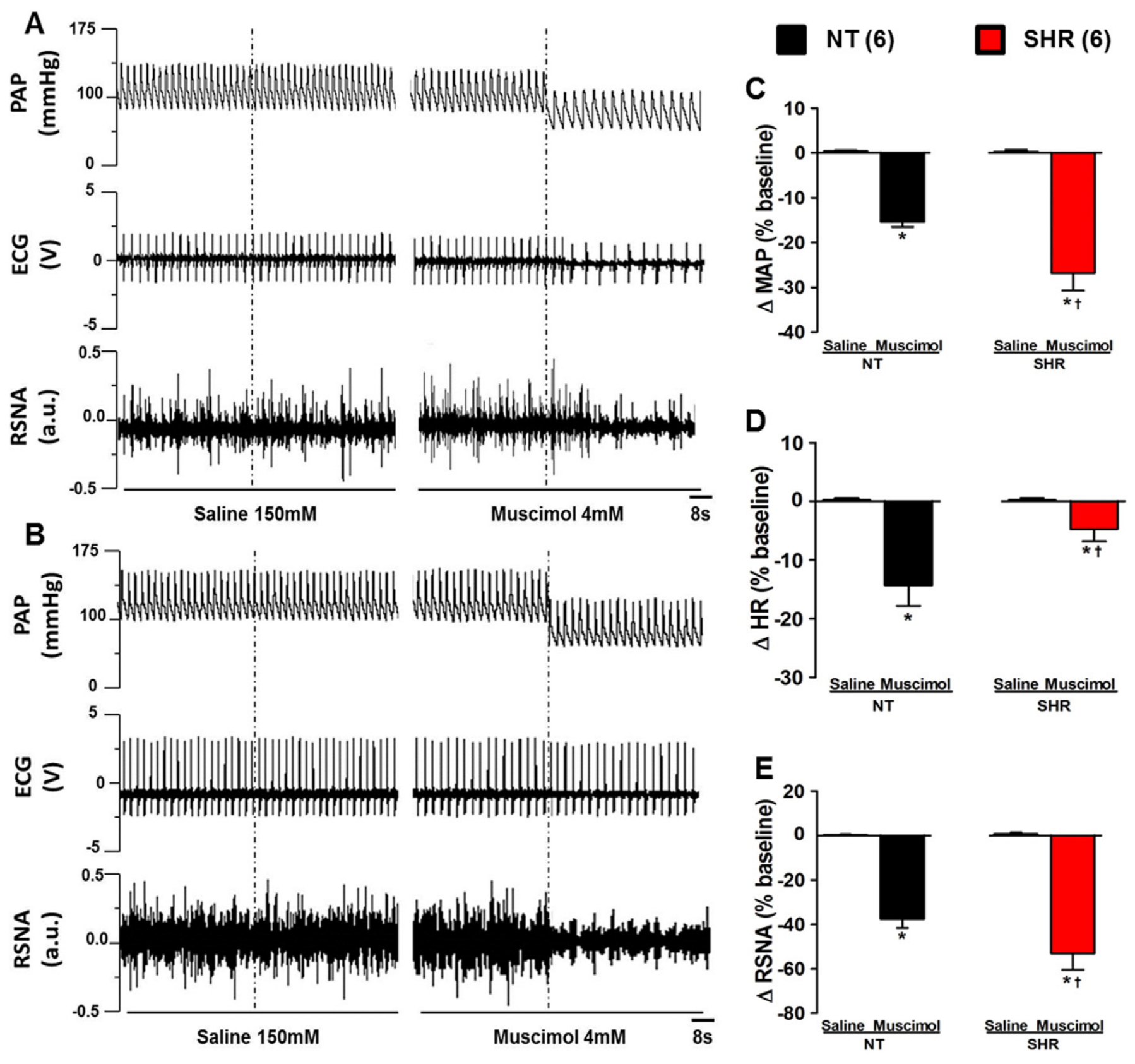

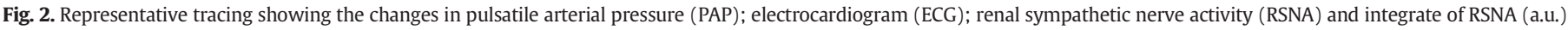

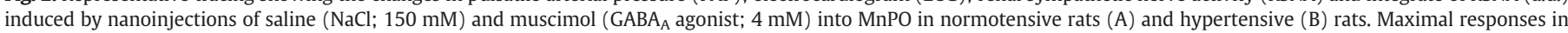

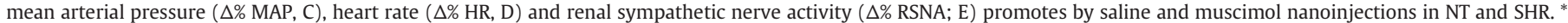
different from the saline nanoinjections; + different from the NT rats; $p<0.05$.

Moreover, all authors have contributed sufficiently in this study to be included as authors. There are no conflicts of interest.

\section{References}

Allen, A.M., 2002. Inhibition of the hypothalamic paraventricular nucleus in spontaneously hypertensive rats dramatically reduces sympathetic vasomotor tone. Hypertension 39, 275-280.

Amaral, N.O., de Oliveira, T.S., Naves, L.M., Filgueira, F.P., Ferreira-Neto, M.L., Schoorlemmer, G.H.M., de Castro, C.H., Freiria-Oliveira, A.H., Xavier, C.H., Colugnati, D.B., Rosa, D.A., Blanch, G.T., Borges, C.L., Soares, C.M.A., Reis, A.A.S., Cravo, S.L. Pedrino, G.R., 2014. Efferent pathways in sodium overload-induced renal vasodilation in rats. PLoS One 9, e109620.

Antunes-Rodrigues, J., de Castro, M., Elias, L.L.K., Valença, M.M., McCann, S.M., 2004. Neuroendocrine control of body fluid metabolism. Physiol. Rev. 84, 169-208.

Blanch, G.T., Freiria-Oliveira, A.H., Murphy, D., Paulin, R.F., Antunes-Rodrigues, J., Colombari, E., Menani, J.V., Colombari, D.S., 2013. Inhibitory mechanism of the nucleus of the solitary tract involved in the control of cardiovascular, dipsogenic, hormonal, and renal responses to hyperosmolality. Am. J. Physiol. Regul. Integr. Comp. Physiol. 304, R531-R542.

Brody, M.J., Johnson, A.K., 1980. Role of the anteroventral third ventricle region in fluid and electrolyte balance, arterial pressure regulation, and hypertension. In: Martini, L, Ganong, WF (Eds.), Frontiers in Neuroendocrinology vol 6. Raven Press, New York, p. 249.

Da Silva, E.F., Freiria-Oliveira, A.H., Custódio, C.H.X., Ghedini, P.C., Bataus, L.A.M., Colombari, E., de Castro, C.H., Colugnati, D.B., Rosa, D.A., Cravo, S.L.D., Pedrino, G.R., 2013. A1 noradrenergic neurons lesions reduce natriuresis and hypertensive responses to hypernatremia in rats. PLoS One 8, e73187.

De Luca, L.A.J., Galaverna, O., Schulkin, J., Yao, S.Z., Epstein, A.N., 1992. The anteroventral wall of the third ventricle and the angiotensinergic component of need-induced sodium intake in the rat. Brain Res. Bull. 28, 73-87.

Gardiner, T.W., Jolley, J.R., Vagnucci, A.H., Stricker, E.M., 1986. Enhanced sodium appetite in rats with lesions centered on nucleus medianus. Behav. Neurosci. 100, 531-535.

Linz, D., Hohl, M., Schutze, J., Mahfoud, F., Speer, T., Linz, B., Hubschle, T., Juretschke, H.P. Dechend, R., Geisel, J., Rutten, H., Bohm, M., 2015. Progression of kidney injury and cardiac remodeling in obese spontaneously hypertensive rats: the role of renal sympathetic innervation. Am. J. Hypertens. 28, 256-265. 
Llewellyn, T., Zheng, H., Liu, X., Xu, B., Patel, K.P., 2012. Median preoptic nucleus and subfornical organ drive renal sympathetic nerve activity via a glutamatergic mechanism within the paraventricular nucleus. Am. J. Physiol. Regul. Integr. Comp. Physiol. 302, R424-R432.

Mangiapane, M.L., Thrasher, T.N., Keil, L.C., Simpson, J.B., Ganong, W.F., 1983. Deficits in drinking and vasopressin secretion after lesions of the nucleus medianus. Neuroendocrinology 37, 73-77.

McKinley, M.J., Johnson, A.K., 2004. The physiological regulation of thirst and fluid intake. News Physiol. Sci. 19, 1-6.

McKinley, M.J., Mathai, M.L., McAllen, R.M., McClear, R.C., Miselis, R.R., Pennington, G.L. Vivas, L., Wade, J.D., Oldfield, B.J., 2004. Vasopressin secretion: osmotic and hormonal regulation by the lamina terminalis. J. Neuroendocrinol. 16, 340-347.

Moreira, T.S., Takakura, A.C., Colombari, E., Menani, J.V., 2009. Antihypertensive effects of central ablations in spontaneously hypertensive rats. Am. J. Physiol. Regul. Integr. Comp. Physiol. 296, R1797-R1806.

Oliveira-Sales, E.B., Toward, M.A., Campos, R.R., Paton, J.F., 2014. Revealing the role of the autonomic nervous system in the development and maintenance of goldblatt hypertension in rats. Auton. Neurosci. 183, 23-29.

Paxinos, G., Watson, C., 1998. The Rat Brain in Stereotaxic Coodinates. Academic Press, Inc., San Diego.

Pedrino, G.R., Monaco, L.R., Cravo, S.L., 2009. Renal vasodilation induced by hypernatraemia: role of alpha-adrenoceptors in the median preoptic nucleus. Clin. Exp. Pharmacol. Physiol. 36, e83-e89.

Pedrino, G.R., Rosa, D.A., Korim, W.S., Cravo, S.L., 2008. Renal sympathoinhibition induced by hypernatremia: involvement of $A 1$ noradrenergic neurons. Auton. Neurosci. 142, $55-63$.
Silveira, L.L., da Silva, E.F., de Andrade, A.M., Xavier, C.H., Freiria-Oliveira, A.H., Colugnati, D.B., de Castro, C.H., Colombari, E., Pedrino, G.R., 2014. Involvement of the median preoptic nucleus in blood pressure control. Neurosci. Lett. 558, 91-96.

Stocker, S.D., Toney, G.M., 2005. Median preoptic neurones projecting to the hypothalamic paraventricular nucleus respond to osmotic, circulating Ang II and baroreceptor input in the rat. J. Physiol. 568, 599-615.

Takeda, K., Nakata, T., Takesako, T., Itoh, H., Hirata, M., Kawasaki, S., Hayashi, J., Oguro, M., Sasaki, S., Nakagawa, M., 1991. Sympathetic inhibition and attenuation of spontaneous hypertension by PVN lesions in rats. Brain Res. 543, 296-300.

Toney, G.M., Stocker, S.D., 2010. Hyperosmotic activation of CNS sympathetic drive: implications for cardiovascular disease. J. Physiol. 588, 3375-3384.

Toney, G.M., Pedrino, G.R., Fink, G.D., Osborn, J.W., 2010. Does enhanced respiratorysympathetic coupling contribute to peripheral neural mechanisms of angiotensin IIsalt hypertension? Exp. Physiol. 95, 587-594.

Tucker, D.C. Saper, C.B. Ruggiero, D.A., Reis, DJ. 1987. Organization of central adrenergic pathways: I. Relationships of ventrolateral medullary projections to the hypothalamus and spinal cord. J. Comp. Neurol. 259, 591-603.

Yasuda, Y., Honda, K., Negoro, H., Higuchi, T., Goto, Y., Fukuda, S., 2000. The contribution of the median preoptic nucleus to renal sympathetic nerve activity increased by intracerebroventricular injection of hypertonic saline in the rat. Brain Res. 867, 107-114. 